\title{
Absorption and the dimensionless overlap integral for two-photon excitation
}

\author{
Gregory J. Fiechtner*,1, James R. Gord \\ Air Force Research Laboratory, Propulsion Directorate, Wright-Patterson Air Force Base, OH 45433-7103, USA
}

Received 12 May 1999; accepted 1 May 2000

\begin{abstract}
The interaction of an absorption line and a resonant laser is often modeled using a spectral overlap integral. Previous investigators have modified the traditional overlap integral to obtain a dimensionless overlap integral that affords a more practical interpretation. Unique to the derivation of the modified integral is the treatment of the laser-spectral-irradiance profile - its area is normalized to equal the spectral full-width at half-maximum. In this paper, the modified laser spectral irradiance is applied to obtain a dimensionless overlap integral for multi-photon absorption, including the case of two-photon absorption. Close attention is given to the units applied to the calculation of absorption rate coefficients, including those for the laser flux, laser irradiance, interaction cross sections, and distribution functions. The results are tabulated for two cases - frequency expressed in units of wave numbers and frequency expressed in units of Hertz. (C) 2001 Elsevier Science Ltd. All rights reserved.
\end{abstract}

Keywords: Spectral overlap; Laser-induced fluorescence; Two-photon spectroscopy

\section{Introduction}

The use of resonant laser absorption processes has become a popular method of making quantitative gas-phase measurements, e.g., measurements of concentrations of minor species (nitric oxide, carbon monoxide, and the hydroxyl radical) in flames [1-3]. In practice, the absorption transition and the laser are not monochromatic, and the overlap integral is applied to take into account the resulting inefficiencies in the absorption process. The overlap integral is typically

\footnotetext{
${ }^{1}$ Present address: Innovative Scientific Solutions, Inc, 2766 Indian Ripple Road, Dayton, OH 45440-3638, USA.

* Corresponding author. Tel.: 1-937-252-2706; fax: 1-937-255-3139.

E-mail address: gjfiech@ward.appl.wpafb.af.mil (G.J. Fiechtner).
} 
defined as the spectral integration over all frequencies of the product of the spectral distribution functions of the laser and of the absorption line. These distribution functions are normalized by forcing their respective areas to equal unity. The resulting overlap integral has units of centimeters. Noting the ambiguous physical meaning of this traditional form of the overlap integral, Partridge and Laurendeau [4,5] developed a dimensionless equivalent which they applied to spectral computations. The modified overlap integral, as noted by these investigators, can be interpreted as the ratio of the total photon-absorption rate coefficient to the ideal photonabsorption rate coefficient that would result for a monochromatic absorber interacting with a monochromatic laser. In deriving their modified overlap integral, these investigators normalized the spectral-irradiance function of the laser by forcing its integral over all frequencies to equal the full-width at half-maximum (FWHM) of the distribution. The resulting integrated laser irradiance was expressed in terms of parameters that are measured in common laboratory experiments.

Partridge and Laurendeau treated the case of one-photon absorption; however, two-photon absorption is required for concentration measurements of a number of different species in reacting flows. For many light atoms and molecules such as $\mathrm{CO}$, the lowest lying excited states can be resonantly connected to the ground state with single photons of vacuum-ultraviolet energy. Hence, for visible fluorescence detection, two-photon excitation is necessary to populate a state that is separated from a lower level by a suitable energy. A number of measurements have utilized two-photon excitation for detecting species such as $\mathrm{N}, \mathrm{O}, \mathrm{C}, \mathrm{H}$, and $\mathrm{CO}$ using subsequent fluorescence [6-15] or amplified spontaneous emission [10,16,17]. Two-photon absorption often populates levels near the ionization continuum; as a result, a third photon from the laser can ionize the molecules. Collection of either electrons or ions in such cases results in impressive limits of detection [18,19]. In this study, we extend the analysis of Partridge and Laurendeau to obtain a dimensionless overlap integral for two-photon absorption.

A number of two-photon spectroscopic models are documented in the literature. In some cases, a chosen function is normalized in such a way that the area equals unity; these cases include the spectral profile of the laser, the autocorrelation of the spectral profile of the laser, and the overlap integral. The overlap integral is often ignored when assuming that the molecular bandwidth is much larger than the bandwidth of the laser. In this study, we consider the application of a dimensionless approach for two-photon absorption that is analogous to the method of Partridge and Laurendeau. In many applications - particularly in flames - the laser photons can be absorbed by more than one process. For instance, two different species (such as $\mathrm{NO}$ and $\mathrm{NO}_{2}, \mathrm{CO}$ and $\mathrm{NH}_{3}$, or $\mathrm{NO}$ and $\mathrm{O}$ ) [20-24] can absorb photons from the same laser, as can two different transitions of the same molecule $[3,7,18,19]$. In these cases it would be convenient if the overlap fractions for both absorbers were expressed on a dimensionless basis using the same normalization procedure. We consider this possibility in the present investigation.

A variety of approaches can be taken in the treatment of dimensions. For example, literature values of the two-photon "cross section" have been reported with units of centimeters raised to the fourth power, centimeters raised to the fourth power divided by watts, centimeters raised to the fourth power divided by seconds, and centimeters raised to the fourth power multiplied by seconds. Therefore, Table 1 containing symbols, their definitions, and units was constructed.

Mathematical details appear in Appendices A-C. Because the two-photon developments are analogous to those for an equivalent one-photon derivation, one-photon absorption calculations 
Table 1

Nomenclature used in the examination of the overlap integral. The second column contains units corresponding to the case in which frequency is expressed in wave numbers. The third column contains units corresponding to the case where frequency is expressed in Hertz

\begin{tabular}{|c|c|c|c|}
\hline \multirow[t]{2}{*}{ Symbol } & \multicolumn{2}{|c|}{ Frequency representation } & \multirow[t]{2}{*}{ Description } \\
\hline & Wave numbers & Hertz & \\
\hline$A_{\mathrm{L}}$ & $\mathrm{cm}^{2}$ & $\mathrm{~cm}^{2}$ & Cross-sectional area of laser \\
\hline$c$ & $\mathrm{~cm} \mathrm{~s}^{-1}$ & $\mathrm{~cm} \mathrm{~s}^{-1}$ & Speed of light \\
\hline$E_{\mathrm{L}}$ & $\mathrm{J}$ & $\mathrm{J}$ & Energy per laser pulse \\
\hline$f(v)$ & $\mathrm{cm}$ & s & Traditional spectral lineshape function of laser \\
\hline$f_{\mathrm{PL}}(v)$ & Dimensionless & Dimensionless & Laser spectral shape function, Partridge and Laurendeau \\
\hline$g(v)$ & $\mathrm{cm}$ & s & Spectral lineshape function of absorber \\
\hline$G^{\langle 2\rangle}(0)$ & Dimensionless & Dimensionless & Second-order intensity correlation function at zero delay \\
\hline$G^{\langle n\rangle}(0)$ & Dimensionless & Dimensionless & $n$ th-order intensity correlation function at zero delay \\
\hline$h$ & $\mathrm{~J}$ s & $\mathrm{J}$ s & Planck's constant \\
\hline$I(v)$ & $\mathrm{W} \mathrm{cm}^{-1}$ & $\mathrm{~J} \mathrm{~cm}^{-2}$ & Spectral irradiance of laser \\
\hline$I_{0}$ & $\mathrm{~W} \mathrm{~cm}^{-2}$ & $\mathrm{~W} \mathrm{~cm}^{-2}$ & Integrated irradiance of laser \\
\hline$I_{0, \mathrm{PL}}$ & $\mathrm{W} \mathrm{cm}^{-1}$ & $\mathrm{~J} \mathrm{~cm}^{-2}$ & Integrated laser irradiance, Partridge and Laurendeau \\
\hline$N$ & $\mathrm{~cm}^{-3}$ & $\mathrm{~cm}^{-3}$ & Number density \\
\hline$\Delta t_{\mathrm{L}}$ & s & s & Temporal pulsewidth of laser \\
\hline$W(v)$ & $\mathrm{cm} \mathrm{s}^{-1}$ & Dimensionless & Spectral absorption rate coefficient \\
\hline$W_{0}$ & $\mathrm{~s}^{-1}$ & $\mathrm{~s}^{-1}$ & Integrated absorption rate coefficient \\
\hline$z$ & $\mathrm{~cm}$ & $\mathrm{~cm}$ & Position \\
\hline$\Gamma^{\langle 1\rangle}$ & $\mathrm{cm}$ & s & Traditional one-photon overlap integral \\
\hline$\Gamma^{\langle n\rangle}$ & $\mathrm{cm}$ & $\mathrm{s}$ & Traditional $n$-photon overlap integral \\
\hline$\Gamma_{\mathrm{PL}}^{\langle 1\rangle}$ & Dimensionless & Dimensionless & One-photon overlap integral, Partridge and Laurendeau \\
\hline$\Gamma_{\mathrm{PL}}^{\langle n\rangle}$ & $\mathrm{cm}^{-(n-1)}$ & $\mathrm{S}^{-(n-1)}$ & $n$-photon overlap integral, Partridge and Laurendeau \\
\hline$\hat{\Gamma}^{\langle 1\rangle}$ & Dimensionless & Dimensionless & Modified traditional one-photon overlap integral \\
\hline$\hat{\Gamma}^{\langle n\rangle}$ & Dimensionless & Dimensionless & Modified traditional $n$-photon overlap integral \\
\hline$\hat{\Gamma}_{\mathrm{PL}}^{\langle 1\rangle}$ & Dimensionless & Dimensionless & Modified one-photon overlap integral, Partridge and Laurendeau \\
\hline$\hat{\Gamma}_{\mathrm{PL}}^{\langle n\rangle}$ & Dimensionless & Dimensionless & Modified $n$-photon overlap integral, Partridge and Laurendeau \\
\hline$\kappa^{\langle 1\rangle}(v)$ & $\mathrm{cm}^{-1}$ & $\mathrm{~cm}^{-1}$ & Spectral absorption coefficient for one-photon absorption \\
\hline$\kappa^{\langle n\rangle}(v)$ & $\mathrm{cm}^{-1}$ & $\mathrm{~cm}^{-1}$ & Spectral absorption coefficient for $n$-photon absorption \\
\hline$v$ & $\mathrm{~cm}^{-1}$ & $\mathrm{~Hz}$ & Frequency \\
\hline$\Delta v_{\mathrm{L}}$ & $\mathrm{cm}^{-1}$ & $\mathrm{~Hz}$ & Spectral bandwidth of laser (FWHM) \\
\hline$\Phi(v)$ & $\mathrm{cm}^{-1} \mathrm{~s}^{-1}$ & $\mathrm{~cm}^{-2}$ & Spectral flux of laser \\
\hline$\Phi_{0}$ & $\mathrm{~cm}^{-2} \mathrm{~s}^{-1}$ & $\mathrm{~cm}^{-2} \mathrm{~s}^{-1}$ & Integrated flux of laser \\
\hline$\Phi_{0, \mathrm{PL}}$ & $\mathrm{cm}^{-1} \mathrm{~s}^{-1}$ & $\mathrm{~cm}^{-2}$ & Integrated laser flux, Partridge and Laurendeau \\
\hline$\sigma^{\langle 1\rangle}(v)$ & $\mathrm{cm}^{2}$ & $\mathrm{~cm}^{2}$ & Spectral cross section for one-photon absorption \\
\hline$\sigma_{0}^{\langle 1\rangle}$ & $\mathrm{cm}$ & $\mathrm{cm}^{2} \mathrm{~s}^{-1}$ & Integrated cross section for one-photon absorption \\
\hline$\sigma^{\langle n\rangle}(v)$ & $\mathrm{cm}^{2}$ & $\mathrm{~cm}^{2}$ & Spectral cross section for $n$-photon absorption \\
\hline$\tilde{\sigma}^{\langle n\rangle}(v)$ & $\mathrm{cm}^{2 n} \mathrm{~S}^{n-1}$ & $\mathrm{~cm}^{2 n} \mathrm{~S}^{n-1}$ & Normalized spectral cross section for $n$-photon absorption \\
\hline$\tilde{\sigma}_{0}^{\langle n\rangle}$ & $\mathrm{cm}^{2 n-1} \mathrm{~s}^{n-1}$ & $\mathrm{~cm}^{2 n} \mathrm{~s}^{n-2}$ & Integrated, normalized cross section for $n$-photon absorption \\
\hline
\end{tabular}

are discussed in Appendix A. Details of two-photon calculations are given in Appendix B. Finally, because of the possible applications of higher-order photon absorption [25-31], the general overlap fraction for $n$-photon absorption is considered in Appendix C. 


\section{Background}

The spectral distribution function can be expressed as the product of a shape function and the strength factor, $S$. The shape function is a dimensionless relation describing the shape of the distribution function, and the strength factor is determined by the method used to normalize the shape function. For example, Partridge and Laurendeau used the example of a Lorentzian distribution function $D(v)$ in terms of the frequency, $v\left(\mathrm{~cm}^{-1}\right)$, expressed as

$$
D(v)=S\left\{1+\left[\frac{2\left(v-v_{0}\right)}{\Delta v}\right]^{2}\right\}^{-1},
$$

where $v_{0}$ denotes the central frequency $\left(\mathrm{cm}^{-1}\right)$ and $\Delta v$ represents the FWHM $\left(\mathrm{cm}^{-1}\right)$. Although we prefer to express frequency in units of wave numbers in this paper, many investigators prefer to express frequency in units of Hertz. The nomenclature appears in Table 1, where the variables are defined along with their units using both conventions (the Hertz-based system is considered in addition to the wave number-based system in Appendices A-C). Traditionally, Eq. (1) is normalized to unity [32,33]; thus, $S=2 /(\pi \Delta v)$, which has units of centimeters. In this example, Eq. (1) can be applied to both the absorption transition and the laser, and the spectral overlap function can then be calculated using the expression

$$
\Gamma^{\langle 1\rangle}=\int_{-\infty}^{\infty} g(v) f(v) \mathrm{d} v,
$$

which has units of centimeters. Here, $g(v)$ and $f(v)$ denote the spectral distribution functions of the absorption line and the laser, respectively $(\mathrm{cm})$, and the superscript $\langle 1\rangle$ denotes one-photon absorption.

Partridge and Laurendeau [4,5] chose to normalize the distribution function of their laser to its FWHM, $\Delta v_{\mathrm{L}}$, such that

$$
\int_{-\infty}^{\infty} f_{\mathrm{PL}}(v) \mathrm{d} v=\Delta v_{\mathrm{L}}
$$

where the subscript PL is used to denote quantities unique to this normalization method. The resulting strength factor is given by $S_{\mathrm{PL}}=2 / \pi$. Consequently, $f_{\mathrm{PL}}(v)$ and $\Gamma_{\mathrm{PL}}^{\langle 1\rangle}$ are dimensionless, and the traditional overlap integral can be obtained by multiplying $\Gamma_{\mathrm{PL}}^{\langle 1\rangle}$ by $\Delta v_{\mathrm{L}}$. The dimensionless form of the overlap integral is equal to the ratio of the total photon absorption rate coefficient in the actual broadened system to that which would exist in the limit of a monochromatic laser interacting with a monochromatic absorption line (for which $\Gamma_{\mathrm{PL}}^{\langle 1\rangle}=1$ ). Hence, the dimensionless form has a simple and practical interpretation. Their spectral normalization scheme is also of practical benefit, which can be observed by writing the spectral irradiance $I(v)\left(\mathrm{W} \mathrm{cm}^{-1}\right)$ as

$$
I(v) \mathrm{d} v=I_{0, \mathrm{PL}} f_{\mathrm{PL}}(v) \mathrm{d} v
$$

and

$$
I_{0, \mathrm{PL}}=\frac{1}{\Delta v_{\mathrm{L}}} \int_{-\infty}^{\infty} I(v) \mathrm{d} v=\frac{E_{\mathrm{L}}}{A_{\mathrm{L}} \Delta v_{\mathrm{L}} \Delta t_{\mathrm{L}}},
$$


where $E_{\mathrm{L}}$ is the energy per laser pulse ( $\left.\mathrm{J}\right)$ (which is commonly measured using a joulemeter), $A_{\mathrm{L}}$ is the cross-sectional area of the laser $\left(\mathrm{cm}^{2}\right)$, and $\Delta t_{\mathrm{L}}$ is the temporal pulsewidth (FWHM) of the laser (s). Therefore, the integrated irradiance, $I_{0, \mathrm{PL}}\left(\mathrm{W} \mathrm{cm}^{-1}\right)$, is expressed in terms of physically measurable parameters. An example of the application of this normalization scheme to one-photon excitation of NO in flames is provided in Ref. [34]. Partridge and Laurendeau [4,5] also discuss similar normalization of the temporal distribution function. Because in this paper we are concerned primarily with the spectral interaction of the laser and the absorbing molecules, the temporal pulseshape is embedded in the integrated irradiance. It is not our intention to imply that the temporal normalization scheme should be neglected; indeed, we often apply both spectral and temporal normalization schemes to our computations [35]. Similarly, the radial profile of the cross-sectional irradiance is neglected in this paper.

In terms of the traditional approach, the absorption rate coefficient can be written as

$$
W_{0}=\sigma_{0}^{\langle 1\rangle} \Phi_{0} \Gamma^{\langle 1\rangle}=\frac{\sigma_{0}^{\langle 1\rangle} I_{0}}{h c v} \Gamma^{\langle 1\rangle},
$$

as noted in Appendix A. Here, $\sigma_{0}^{\langle 1\rangle}$ is the integrated cross section for one-photon absorption (cm), $\Phi_{0}$ is the integrated laser flux $\left(\mathrm{cm}^{-2} \mathrm{~s}^{-1}\right), h$ is the Planck constant $(\mathrm{J} \mathrm{s}), c$ is the speed of light $\left(\mathrm{cm} \mathrm{s}^{-1}\right)$ and $I_{0}$ is the integrated laser irradiance $\left(\mathrm{W} \mathrm{cm}^{-2}\right)$. In terms of the dimensionless overlap integral, the corresponding integrated absorption rate coefficient is expressed as

$$
W_{0}=\sigma_{0}^{\langle 1\rangle} \Phi_{0, \mathrm{PL}} \Gamma_{\mathrm{PL}}^{\langle 1\rangle}=\frac{\sigma_{0}^{\langle 1\rangle} I_{0, \mathrm{PL}}}{h c v} \Gamma_{\mathrm{PL}}^{\langle 1\rangle} .
$$

\section{Approach}

Two-photon absorption involves the simultaneous absorption of two photons, with the restriction that the sum of the photon energies must equal the change in energy between the initial and the final molecular states [36-41]. The two-photon overlap integral can be written as (see Appendix B)

$$
\Gamma^{\langle 2\rangle}=\int_{-\infty}^{\infty} \int_{-\infty}^{\infty} g\left(v_{1}+v_{2}\right) f\left(v_{1}\right) f\left(v_{2}\right) \mathrm{d} v_{1} \mathrm{~d} v_{2}
$$

which has units of centimeters. The two-photon overlap integral can be used to calculate an integrated two-photon absorption rate coefficient using the relation

$$
W_{0}=2 \tilde{\sigma}_{0}^{\langle 2\rangle} \Phi_{0}^{2} G^{\langle 2\rangle}(0) \Gamma^{\langle 2\rangle} .
$$

Here, $\tilde{\sigma}_{0}^{\langle 2\rangle}$ is the integrated, normalized cross section for two-photon absorption $\left(\mathrm{cm}^{3} \mathrm{~s}\right)$ and $G^{\langle 2\rangle}(0)$ is the second-order intensity correlation function at zero delay.

The equivalent expressions for $n$-photon absorption (as shown in Appendix C) are

$$
\Gamma^{\langle n\rangle}=\int_{-\infty}^{\infty} \int_{-\infty}^{\infty} \cdots \int_{-\infty}^{\infty} g\left(v_{1}+v_{2}+\cdots+v_{n}\right)\left[f\left(v_{1}\right) f\left(v_{2}\right) \cdots f\left(v_{n}\right)\right] \mathrm{d} v_{1} \mathrm{~d} v_{2} \cdots \mathrm{d} v_{n}
$$


and

$$
W_{0}=n \tilde{\sigma}_{0}^{\langle n\rangle} G^{\langle n\rangle}(0) \Phi_{0}^{n} \Gamma^{\langle n\rangle},
$$

where $\tilde{\sigma}_{0}^{\langle n\rangle}$ is the integrated, normalized cross section for $n$-photon absorption $\left(\mathrm{cm}^{2 n-1} \mathrm{~s}^{n-1}\right)$ and $G^{\langle n\rangle}(0)$ is the $n$th order intensity correlation function at zero delay. Thus, the overlap integral for $n$-photon absorption has units of centimeters, independent of the value of $n$. In contrast, if the laser spectral profile function of Eqs. (4) and (5) is applied, the resulting absorption rate coefficient is given by

$$
W_{0}=n \tilde{\sigma}_{0}^{\langle n\rangle} G^{\langle n\rangle}(0) \Phi_{0, \mathrm{PL}}^{n} \Gamma_{\mathrm{PL}}^{\langle n\rangle},
$$

where

$$
\begin{aligned}
\Gamma_{\mathrm{PL}}^{\langle n\rangle}= & \int_{-\infty}^{\infty} \int_{-\infty}^{\infty} \cdots \int_{-\infty}^{\infty} g\left(v_{1}+v_{2}+\cdots+v_{n}\right) \\
& \times\left[f_{\mathrm{PL}}\left(v_{1}\right) f_{\mathrm{PL}}\left(v_{2}\right) \cdots f_{\mathrm{PL}}\left(v_{n}\right)\right] \mathrm{d} v_{1} \mathrm{~d} v_{2} \cdots \mathrm{d} v_{n}
\end{aligned}
$$

which has units of $\mathrm{cm}^{-(n-1)}$. Therefore, the simple and practical nature of a dimensionless overlap fraction is lost in the case of multi-photon absorption.

Often encountered in spectroscopic applications is the case in which the FWHM bandwidth of the absorber is substantially larger than that of the laser. The overlap integral can then be simplified by holding $g\left(v_{1}+v_{2}+\cdots+v_{n}\right)$ constant at $n$ times the centerline frequency of the laser $\left(v_{\mathrm{L}, 0}\right)$; this results in the approximate expression for the overlap integral, based on the normalization method of Partridge and Laurendeau,

$$
\Gamma_{\mathrm{PL}}^{\langle n\rangle} \cong g\left(n v_{\mathrm{L}, 0}\right) \int_{-\infty}^{\infty} \int_{-\infty}^{\infty} \cdots \int_{-\infty}^{\infty}\left[f_{\mathrm{PL}}\left(v_{1}\right) f_{\mathrm{PL}}\left(v_{2}\right) \cdots f_{\mathrm{PL}}\left(v_{n}\right)\right] \mathrm{d} v_{1} \mathrm{~d} v_{2} \cdots \mathrm{d} v_{n}
$$

and the equivalent approximate expression using the traditional normalization routine,

$$
\Gamma^{\langle n\rangle} \cong g\left(n v_{\mathrm{L}, 0}\right) \int_{-\infty}^{\infty} \int_{-\infty}^{\infty} \cdots \int_{-\infty}^{\infty}\left[f\left(v_{1}\right) f\left(v_{2}\right) \cdots f\left(v_{n}\right)\right] \mathrm{d} v_{1} \mathrm{~d} v_{2} \cdots \mathrm{d} v_{n} .
$$

Eqs. (14) and (15) highlight two approaches to placing the overlap integral on a dimensionless basis. In Eq. (14), the quantity outside the integral has units of centimeters, while the remaining quantity has units of $\mathrm{cm}^{-n}$. Therefore, it is desirable to define a dimensionless quantity given by

$$
\hat{\Gamma}_{\mathrm{PL}}^{\langle n\rangle}=\left(\frac{1}{\Delta v_{\mathrm{L}}}\right)^{n-1} \Gamma_{\mathrm{PL}}^{\langle n\rangle}
$$

for which the integrated absorption rate coefficient is

$$
W_{0}=n \tilde{\sigma}_{0}^{\langle n\rangle} G^{n}(0) \Phi_{0, \mathrm{PL}}^{n} \Delta v_{\mathrm{L}}^{n-1} \hat{\Gamma}_{\mathrm{PL}}^{\langle n\rangle} .
$$

Because we often apply the normalization of Partridge and Laurendeau in one-photon spectral computations, we prefer this normalization scheme when computing two-photon absorption spectra. 
A different approach can be found from examining Eq. (15), where it is obvious that the integrated quantity is already dimensionless, regardless of the number of photons absorbed. This suggests the definition of two new variables

$$
\hat{g}(v)=\Delta v_{\mathrm{C}} g(v),
$$

and a modified (and dimensionless) overlap integral

$$
\hat{\Gamma}^{\langle n\rangle}=\int_{-\infty}^{\infty} \int_{-\infty}^{\infty} \cdots \int_{-\infty}^{\infty} \hat{g}\left(v_{1}+v_{2}+\cdots+v_{n}\right)\left[f\left(v_{1}\right) f\left(v_{2}\right) \cdots f\left(v_{n}\right)\right] \mathrm{d} v_{1} \mathrm{~d} v_{2} \cdots \mathrm{d} v_{n}
$$

which can be inserted into Eq. (11) as a direct replacement of the quantity $\Gamma^{\langle n\rangle}$. Here, $\Delta v_{\mathrm{C}}\left(\mathrm{cm}^{-1}\right)$ is a parameter describing the broadening of the absorbing medium, which, for example, could represent collisional broadening. A potential disadvantage of this scheme is that treatment of the laser irradiance afforded by Eqs. (4) and (5) is neglected in favor of the traditional normalization method.

\section{Conclusions}

For a one-photon absorption process, Partridge and Laurendeau have shown that a dimensionless overlap integral is of practical value for spectral computations. Although a dimensionless two-photon integral would be of equal utility, it cannot be obtained directly using their modified laser spectral distribution. We have developed two alternative methods for obtaining dimensionless overlap integrals for multi-photon absorption. First, a dimensionless equivalent for $n$-photon absorption can be obtained through multiplication by the spectral bandwidth raised to the power $n-1$. Because historically we apply the normalization scheme of Partridge and Laurendeau to one-photon calculations, we prefer this method for our present calculations of two-photon spectra. Alternatively, a dimensionless spectral distribution function for the absorber can be obtained by multiplying the dimensional quantity by the spectral FWHM bandwidth. Finally, careful attention has been given to the units that should be applied for each variable. Table 1 containing the variables and dimensions is useful when calculating two-photon-absorption spectra and also for interpreting the literature values for absorption cross sections.

\section{Acknowledgements}

This work was supported by US Air Force Contracts F33615-95-C-2507 and F33625-98-C-2858 and by the US Department of Energy, Office of Basic Energy Sciences. The authors thank Marian M. Whitaker for editorial assistance.

\section{Appendix A. One-photon absorption}

Development of an expression for the dimensionless two-photon overlap fraction is analogous to that for one-photon absorption. Therefore, the absorption rate coefficient for one-photon absorp- 
tion will be reviewed in this appendix. Consider the attenuation of the spectral irradiance $(\mathrm{W} \mathrm{cm})$ in a frequency interval $\mathrm{d} v\left(\mathrm{~cm}^{-1}\right)$ propagating in the $z(\mathrm{~cm})$ direction [42],

$$
\frac{1}{I(v) \mathrm{d} v} \frac{\mathrm{d} I(v) \mathrm{d} v}{\mathrm{~d} z}=-\kappa^{\langle 1\rangle}(v)
$$

where $\kappa^{\langle 1\rangle}(v)$ is the spectral absorption coefficient $\left(\mathrm{cm}^{-1}\right)$, and the superscript $\langle 1\rangle$ is applied to denote a one-photon absorption process. Since $\kappa^{\langle 1\rangle}(v)$ is proportional to the number density of ground-state molecules $N\left(\mathrm{~cm}^{-3}\right)$, we can write

$$
\kappa^{\langle 1\rangle}(v)=N \sigma^{\langle 1\rangle}(v),
$$

where $\sigma^{\langle 1\rangle}(v)$ is the spectral absorption cross section $\left(\mathrm{cm}^{2}\right)$. Eqs. (A.1) and (A.2) apply when expressing frequency in units of wave numbers or Hertz. The spectral absorption cross section contains spectral broadening information concerning the absorbing molecules and can be written

$$
\sigma^{\langle 1\rangle}(v)=\sigma_{0}^{\langle 1\rangle} g(v),
$$

where $g(v)$ is traditionally normalized such that

$$
\int_{-\infty}^{\infty} g(v) \mathrm{d} v=1
$$

Therefore, for frequency expressed in wave numbers, $g(v)$ has units of centimeters; for frequency expressed in Hertz, $g(v)$ has units of seconds. The integrated absorption cross section, $\sigma_{0}^{\langle 1\rangle}$, has units of either centimeters or $\mathrm{cm}^{2} \mathrm{~s}$, respectively, for frequency expressed in wave numbers or Hertz, and is related to the spectral absorption cross section by the equation

$$
\sigma_{0}^{\langle 1\rangle}=\int_{-\infty}^{\infty} \sigma^{\langle 1\rangle}(v) \mathrm{d} v .
$$

The cross section can be used to calculate the absorption rate coefficient, which, on a spectral basis, takes the form

$$
W(v) \mathrm{d} v=\sigma(v) \frac{I(v) \mathrm{d} v}{h c v},
$$

where $I(v)$ is the spectral laser irradiance with units of $\mathrm{W} \mathrm{cm}^{-1}$ when frequency is expressed in wave numbers and $\mathrm{J} \mathrm{cm}^{-2}$ when frequency is expressed in Hertz. From Eq. (A.6), we find that $W(v)$ has units of $\mathrm{cm} \mathrm{s}^{-1}$ when frequency is expressed in wave numbers. However, when frequency is expressed in Hertz, Eq. (A.6) is replaced by the expression

$$
W\left(v_{\mathrm{Hz}}\right) \mathrm{d} v_{\mathrm{Hz}}=\sigma\left(v_{\mathrm{Hz}}\right) \frac{I\left(v_{\mathrm{Hz}}\right) \mathrm{d} v_{\mathrm{Hz}}}{h v_{\mathrm{Hz}}},
$$

and $W\left(v_{\mathrm{Hz}}\right)$ is dimensionless. To eliminate the need for two expressions describing the spectral absorption rate coefficient, we can write

$$
W(v) \mathrm{d} v=\sigma(v) \Phi(v) \mathrm{d} v,
$$


where $\Phi(v)$ is the spectral flux, which is obtained by dividing the spectral irradiance by the energy per photon. The spectral flux has units of $\mathrm{cm}^{-1} \mathrm{~s}^{-1}$ or $\mathrm{cm}^{-2}$ when frequency is expressed in wave numbers or Hertz, respectively.

The laser spectral shape function is traditionally normalized such that its area is unity, as indicated by the expression

$$
\int_{-\infty}^{\infty} f(v) \mathrm{d} v=1
$$

Thus, $f(v)$ has units of centimeters when frequency is expressed in wave numbers and units of seconds when frequency is expressed in Hertz. We can then write

$$
I(v)=I_{0} f(v),
$$

where $I_{0}$ is the integrated laser irradiance $\left(\mathrm{W} \mathrm{cm}^{-2}\right)$ given by

$$
I_{0}=\int_{-\infty}^{\infty} I(v) \mathrm{d} v
$$

In the same manner, we can write

$$
\Phi(v)=\Phi_{0} f(v),
$$

where $\Phi_{0}$ is the integrated laser flux $\left(\mathrm{cm}^{-2} \mathrm{~s}^{-1}\right)$, which can be expressed as

$$
\Phi_{0}=\int_{-\infty}^{\infty} \Phi(v) \mathrm{d} v .
$$

Integration of Eq. (A.8) over all frequencies yields the integrated absorption rate coefficient $\left(\mathrm{s}^{-1}\right)$, which is given by

$$
W_{0}=\sigma_{0}^{\langle 1\rangle} \Phi_{0} \int_{-\infty}^{\infty} f(v) g(v) \mathrm{d} v
$$

Substitution of Eq. (2) into Eq. (A.14) yields the rate coefficient

$$
W_{0}=\sigma_{0}^{\langle 1\rangle} \Phi_{0} \Gamma^{\langle 1\rangle},
$$

where $\Gamma^{\langle 1\rangle}$ has units of centimeters when frequency is expressed in wave numbers and units of seconds when frequency is expressed in Hertz.

\section{Appendix B. Two-photon absorption}

Consider the absorption of two photons, $v_{1}$ and $v_{2}$, subject to the condition that the quantity $2 h c\left(v_{1}+v_{2}\right)$ corresponds to the energy difference between two states that are coupled by a twophoton transition $\left(2 h v_{1}+2 h v_{2}\right.$ when frequency is expressed in Hertz). The attenuation of the spectral irradiance in the frequency intervals $\mathrm{d} v_{1}$ and $\mathrm{d} v_{2}$ can then be written as a pair of coupled 
differential equations [42-48],

$$
\frac{1}{I\left(v_{1}\right) \mathrm{d} v_{1}} \frac{\mathrm{d} I\left(v_{1}\right) \mathrm{d} v_{1}}{\mathrm{~d} z}=-\kappa^{\langle 2\rangle}\left(v_{2}, v_{1}\right)
$$

and

$$
\frac{1}{I\left(v_{2}\right) \mathrm{d} v_{2}} \frac{\mathrm{d} I\left(v_{2}\right) \mathrm{d} v_{2}}{\mathrm{~d} z}=-\kappa^{\langle 2\rangle}\left(v_{1}, v_{2}\right),
$$

where the superscript $\langle 2\rangle$ is used to denote a two-photon absorption process. The coefficient $\kappa^{\langle 2\rangle}(v)\left(\mathrm{cm}^{-1}\right)$ is analogous to the spectral one-photon absorption coefficient. Therefore, we can write

$$
\kappa^{\langle 2\rangle}\left(v_{1}, v_{2}\right)=N \sigma^{\langle 2\rangle}\left(v_{1}, v_{2}\right)
$$

and

$$
\kappa^{\langle 2\rangle}\left(v_{2}, v_{1}\right)=N \sigma^{\langle 2\rangle}\left(v_{2}, v_{1}\right),
$$

where $\sigma^{\langle 2\rangle}(v)$ is the spectral two-photon-absorption cross section $\left(\mathrm{cm}^{2}\right)$. Unlike in the case of one-photon absorption, the spectral two-photon-absorption cross section that describes the absorption of a photon in the frequency interval $\mathrm{d} v_{1}$ depends on the spectral irradiance in the frequency interval $\mathrm{d} v_{2}$, and the absorption of a photon in the frequency interval $\mathrm{d} v_{2}$ depends on the spectral irradiance in the frequency interval $\mathrm{d} v_{1}$. Therefore, the functional representation of the absorption coefficients given in Eqs. (B.3) and (B.4) reflects this interdependence through the laser frequency with reversed subscripts 1 and 2 . Hence, we may write

$$
\sigma^{\langle 2\rangle}\left(v_{1}, v_{2}\right)=\tilde{\sigma}^{\langle 2\rangle}\left(v_{1}+v_{2}\right) G^{\langle 2\rangle}(0) \Phi\left(v_{1}\right) \mathrm{d} v_{1}
$$

and

$$
\sigma^{\langle 2\rangle}\left(v_{2}, v_{1}\right)=\tilde{\sigma}^{\langle 2\rangle}\left(v_{1}+v_{2}\right) G^{\langle 2\rangle}(0) \Phi\left(v_{2}\right) \mathrm{d} v_{2},
$$

where $\tilde{\sigma}^{\langle 2\rangle}\left(v_{1}+v_{2}\right)$ is the normalized spectral absorption cross section $\left(\mathrm{cm}^{4} \mathrm{~s}\right)$ [47], and $G^{\langle 2\rangle}(0)$ is the dimensionless second-order intensity correlation function at delay zero [38,45-47,49-52]. The normalized spectral cross section can, in turn, be written as

$$
\tilde{\sigma}^{\langle 2\rangle}\left(v_{1}+v_{2}\right)=\tilde{\sigma}_{0}^{\langle 2\rangle} g\left(v_{1}+v_{2}\right),
$$

where $\tilde{\sigma}_{0}^{\langle 2\rangle}$ is the integrated, normalized absorption cross section, with units of $\mathrm{cm}^{3} \mathrm{~s}$ when frequency is expressed in wave numbers and units of $\mathrm{cm}^{4}$ when frequency is expressed in Hertz [52-63]. The integrated, normalized absorption cross section can be written as

$$
\tilde{\sigma}_{0}^{\langle 2\rangle}=\int_{-\infty}^{\infty} \tilde{\sigma}^{\langle 2\rangle}(v) \mathrm{d} v
$$

Analogous to the one-photon treatment of the spectral absorption rate coefficient, we can write

$$
W\left(v_{1}\right) \mathrm{d} v_{1}=\sigma^{\langle 2\rangle}\left(v_{1}+v_{2}\right) \Phi\left(v_{1}\right) \mathrm{d} v_{1}
$$


and

$$
W\left(v_{2}\right) \mathrm{d} v_{2}=\sigma^{\langle 2\rangle}\left(v_{1}+v_{2}\right) \Phi\left(v_{2}\right) \mathrm{d} v_{2}
$$

Since one photon is absorbed from each of the frequency intervals $\mathrm{d} v_{1}$ and $\mathrm{d} v_{2}$, we can combine Eqs. (B.9) and (B.10) and apply Eqs. (A.12) and (B.7) to obtain

$$
W(v) \mathrm{d} v=2 \tilde{\sigma}_{0}^{\langle 2\rangle} \Phi_{0}^{2} G^{\langle 2\rangle}(0) g\left(v_{1}+v_{2}\right) f\left(v_{1}\right) f\left(v_{2}\right) \mathrm{d} v_{1} \mathrm{~d} v_{2} .
$$

Integration of Eq. (B.11) over all frequencies yields the integrated absorption rate coefficient, which is given by

$$
W_{0}=2 \tilde{\sigma}_{0}^{\langle 2\rangle} \Phi_{0}^{2} G^{\langle 2\rangle}(0) \int_{-\infty}^{\infty} \int_{-\infty}^{\infty} g\left(v_{1}+v_{2}\right) f\left(v_{1}\right) f\left(v_{2}\right) \mathrm{d} v_{1} \mathrm{~d} v_{2} .
$$

As in Appendix A for the case of one-photon absorption, we can now define the spectral overlap integral for two-photon absorption as

$$
\Gamma^{\langle 2\rangle}=\int_{-\infty}^{\infty} \int_{-\infty}^{\infty} g\left(v_{1}+v_{2}\right) f\left(v_{1}\right) f\left(v_{2}\right) \mathrm{d} v_{1} \mathrm{~d} v_{2} .
$$

The resulting integrated absorption rate coefficient is then written as

$$
W_{0}=2 \tilde{\sigma}_{0}^{\langle 2\rangle} \Phi_{0}^{2} G^{\langle 2\rangle}(0) \Gamma^{\langle 2\rangle} .
$$

Note that the spectral overlap integral for two-photon absorption has units of centimeters when frequency is expressed in wave numbers and units of seconds when frequency is expressed in Hertz. We can then write Eq. (B.13) as $[9,46,62,64]$

$$
\Gamma^{\langle 2\rangle}=\int_{-\infty}^{\infty} g\left(2 v_{\mathrm{L}}\right) h\left(2 v_{\mathrm{L}}\right) \mathrm{d}\left(2 v_{\mathrm{L}}\right)
$$

where

$$
h\left(2 v_{\mathrm{L}}\right)=\int_{-\infty}^{\infty} f\left(2 v_{\mathrm{L}}-v_{2}\right) f\left(v_{2}\right) \mathrm{d} v_{2}
$$

is the autocorrelation of the laser spectral profile. Here, $v_{\mathrm{L}}$ is the laser frequency and $v_{\mathrm{L}, 0}$ denotes the central frequency of the laser spectral profile, which is tuned to coincide with half the central frequency of the absorber. If we assume the laser shape function to be Gaussian, such that

$$
f\left(v_{\mathrm{L}}\right)=\frac{2}{\Delta v_{\mathrm{L}}} \sqrt{\frac{\ln 2}{2 \pi}} \exp \left[-4 \ln 2\left(\frac{v_{\mathrm{L}}-v_{\mathrm{L}, 0}}{\Delta v_{\mathrm{L}}}\right)^{2}\right]
$$

then substitution of Eq. (B.17) into Eq. (B.16) yields the distribution

$$
h\left(2 v_{\mathrm{L}}\right)=\frac{2}{\Delta v_{\mathrm{L}}} \sqrt{\frac{\ln 2}{2 \pi}} \exp \left[-4 \ln 2\left(\frac{2 v_{\mathrm{L}}-2 v_{\mathrm{L}, 0}}{\sqrt{2} \Delta v_{\mathrm{L}}}\right)^{2}\right] .
$$


Thus, the laser spectral profile has an effective bandwidth for two-photon absorption that is $\sqrt{2}$ times greater than that for one-photon absorption [9,56,60,65-69].

If the spectral bandwidth of the molecular absorber is much wider than that of the laser $[36,39,70-73]$, the molecular spectral profile function can be evaluated at twice the centerline frequency of the laser and taken outside the overlap integral, as demonstrated in Eq. (15). This situation is often encountered in spectral studies of the condensed phase in which the quantity $\tilde{\sigma}_{0}^{\langle 2\rangle} g\left(2 v_{\mathrm{L}, 0}\right)$ can be reported in units of "GM" [74-76] (in honor of Maria Göppert-Mayer, who first documented the concept of two-photon absorption) [77]. Note that units of GM apply only when frequency is expressed in Hertz.

\section{Appendix C. Multiphoton absorption}

For absorption of $n$ photons, we can write the following set of $n$ coupled differential equations $[47,78]$

$$
\begin{gathered}
\frac{1}{I\left(v_{1}\right) \mathrm{d} v_{1}} \frac{\mathrm{d} I\left(v_{1}\right) \mathrm{d} v_{1}}{\mathrm{~d} z}=-\kappa^{\langle n\rangle}\left(v_{1}, v_{2}, v_{3}, v_{4}, \ldots, v_{n}\right), \\
\frac{1}{I\left(v_{2}\right) \mathrm{d} v_{2}} \frac{\mathrm{d} I\left(v_{2}\right) \mathrm{d} v_{2}}{\mathrm{~d} z}=-\kappa^{\langle n\rangle}\left(v_{2}, v_{1}, v_{3}, v_{4}, \ldots, v_{n}\right), \\
\vdots \\
\frac{1}{I\left(v_{n}\right) \mathrm{d} v_{n}} \frac{\mathrm{d} I\left(v_{n}\right) \mathrm{d} v_{n}}{\mathrm{~d} z}=-\kappa^{\langle n\rangle}\left(v_{n}, v_{1}, v_{2}, v_{3}, \ldots, v_{n-1}\right),
\end{gathered}
$$

where the spectral absorption coefficients can are written as

$$
\begin{aligned}
\kappa^{\langle n\rangle}\left(v_{1}, v_{2}, v_{3}, v_{4}, \ldots, v_{n}\right)= & N \sigma^{\langle n\rangle}\left(v_{1}, v_{2}, v_{3}, v_{4}, \ldots, v_{n}\right), \\
\kappa^{\langle n\rangle}\left(v_{2}, v_{1}, v_{3}, v_{4}, \ldots, v_{n}\right)= & N \sigma^{\langle n\rangle}\left(v_{2}, v_{1}, v_{3}, v_{4}, \ldots, v_{n}\right), \\
\vdots & \\
\kappa^{\langle n\rangle}\left(v_{n}, v_{1}, v_{2}, v_{3}, \ldots, v_{n-1}\right)= & N \sigma^{\langle n\rangle}\left(v_{n}, v_{1}, v_{2}, v_{3}, \ldots, v_{n-1}\right) .
\end{aligned}
$$

The laser-dependent spectral cross sections $\left(\mathrm{cm}^{2}\right)$ can be expressed in terms of the normalized spectral cross sections by

$$
\begin{aligned}
\sigma^{\langle n\rangle}\left(v_{1}, v_{2}, v_{3}, \ldots, v_{n}\right)= & \tilde{\sigma}^{\langle n\rangle}\left(v_{1}+v_{2}+\cdots+v_{n}\right) G^{\langle n\rangle}(0)\left[\Phi\left(v_{2}\right) \mathrm{d} v_{2} \Phi\left(v_{3}\right) \mathrm{d} v_{3} \cdots \Phi\left(v_{n}\right) \mathrm{d} v_{n}\right], \\
\sigma^{\langle n\rangle}\left(v_{2}, v_{1}, v_{3}, \ldots, v_{n}\right)= & \tilde{\sigma}^{\langle n\rangle}\left(v_{1}+v_{2}+\cdots+v_{n}\right) G^{\langle n\rangle}(0)\left[\Phi\left(v_{1}\right) \mathrm{d} v_{1} \Phi\left(v_{3}\right) \mathrm{d} v_{3} \cdots \Phi\left(v_{n}\right) \mathrm{d} v_{n}\right], \\
& \vdots \\
\sigma^{\langle n\rangle}\left(v_{n}, v_{1}, v_{2}, \ldots, v_{n-1}\right)= & \tilde{\sigma}^{\langle n\rangle}\left(v_{1}+v_{2}+\cdots+v_{n}\right) G^{\langle n\rangle}(0) \\
& \times\left[\Phi\left(v_{1}\right) \mathrm{d} v_{1} \Phi\left(v_{2}\right) \mathrm{d} v_{2} \cdots \Phi\left(v_{n-1}\right) \mathrm{d} v_{n-1}\right],
\end{aligned}
$$

\footnotetext{
${ }^{2}$ The unit GM is equal to $10^{-50} \mathrm{~cm}^{4} \mathrm{~s}$.
} 
where

$$
\tilde{\sigma}^{\langle n\rangle}\left(v_{1}+v_{2}+\cdots+v_{n}\right)=\tilde{\sigma}_{0}^{\langle n\rangle} g\left(v_{1}+v_{2}+\cdots+v_{n}\right) .
$$

Therefore, the normalized spectral cross section for $n$-photon absorption has units of $\mathrm{cm}^{2 n} \mathrm{~s}^{n-1}$. The integrated, normalized cross section for $n$-photon absorption has units of $\mathrm{cm}^{2 n-1} \mathrm{~s}^{n-1}$ when frequency is expressed in wave numbers and units of $\mathrm{cm}^{2 n} \mathrm{~s}^{n-2}$ when frequency is expressed in Hertz $[38,79,80]$. The spectral absorption rate coefficient becomes

$$
W(v) \mathrm{d} v=n \tilde{\sigma}_{0}^{\langle n\rangle} G^{n}(0) \Phi_{0}^{n} g\left(v_{1}+v_{2}+\cdots+v_{n}\right)\left[f\left(v_{1}\right) \mathrm{d} v_{1} f\left(v_{2}\right) \mathrm{d} v_{2} \cdots f\left(v_{n}\right) \mathrm{d} v_{n}\right] .
$$

Integration over all frequencies yields the integrated absorption rate coefficient,

$$
W_{0}=n \tilde{\sigma}_{0}^{\langle n\rangle} G^{\langle n\rangle}(0) \Phi_{0}^{n} \Gamma^{\langle n\rangle},
$$

where the spectral-overlap function for $n$-photon absorption has been defined as

$$
\Gamma^{\langle n\rangle}=\int_{-\infty}^{\infty} \int_{-\infty}^{\infty} \cdots \int_{-\infty}^{\infty} g\left(v_{1}+v_{2}+\cdots+v_{n}\right)\left[f\left(v_{1}\right) f\left(v_{2}\right) \cdots f\left(v_{n}\right)\right] \mathrm{d} v_{1} \mathrm{~d} v_{2} \cdots \mathrm{d} v_{n} .
$$

Fast fluctuations in laser irradiance are taken into account by application of the $n$ th-order correlation function $G^{\langle n\rangle}(0)[38,49,50,65]$.

\section{References}

[1] Carter CD, Barlow RS. Opt Lett 1994;19:299.

[2] Barlow RS, Fiechtner GJ, Chen J-Y. 26th International Symposium on Combustion. The Combustion Institute, Pittsburgh, PA, pp. 2199-2205, 1996.

[3] Barlow RS, Frank JH. Twenty-Seventh Symposium (International) on Combustion. Pittsburgh, PA: The Combustion Institute, 1998. p. 1087-95.

[4] Partridge Jr. WP, Laurendeau NM. Appl Opt 1995;34:2645.

[5] Partridge Jr. WP. Experimental assessment and enhancement of planar laser-induced fluorescence measurements of nitric oxide in an inverse diffusion flame. Ph.D. dissertation, Purdue University, West Lafayette, Indiana, 1995.

[6] Salmon JT, Laurendeau NM. Appl Opt 1987;26:2881.

[7] Seitzman JM, Haumann J, Hanson RK. Appl Opt 1987;26:2892.

[8] Westblom U, Aldén M. Appl Spectrosc 1990;44:881.

[9] Agrup S. Measurements of quenched fluorescence lifetimes and stimulated emission from flame radicals. Ph.D. dissertation, Lund Institute of Technology, Lund, Sweden, 1994.

[10] Kohse-Höinghaus K. Prog Energy Combust Sci 1994;20:203.

[11] Ganguly BN, Bletzinger P. J Appl Phys 1997;82:4772.

[12] Snyder SC, LeRoux AM. IEEE Trans Plasma Sci 1997;25:824.

[13] Tserepi AD, Wurzberg E, Miller TA. Chem Phys Lett 1997;265:297.

[14] Adams SF, Miller TA. Chem Phys Lett 1998;295:305.

[15] Reeves M, Musculus M, Farrell P. Appl Opt 1998;37:6627.

[16] Georgiev N, Nyholm K, Fritzon R, Aldén M. Opt Commun 1994;108:71.

[17] Brown MS, Jeffries JB. Appl Opt 1995;34:1127.

[18] Hurst GS, Payne MG, Kramer SD, Young JP. Rev Mod Phys 1979;51:767.

[19] Ashfold MNR, Clement SG, Howe JD, Western CW. J Chem Soc Faraday Trans 1993;89:1153.

[20] Aldén M, Edner H, Wallin S. Opt Lett 1985;10:529.

[21] Westblom U, Aldén M. Appl Opt 1990;29:4844.

[22] Nyholm K, Fritzon R, Gorgiev N, Aldén M. Opt Commun 1995;114:76. 
[23] Östmark H, Carlson M, Ekvall K. Combust Flame 1996;105:381.

[24] Georgiev N, Aldén M. Spectrochim Acta B 1997;52:1105.

[25] Tjossem PJH, Cool TA. Chem Phys Lett 1983;100:479.

[26] Tjossem PJH, Cool TA. 20th International Symposium on Combustion. Pittsburgh, PA; The Combustion Institute, 1984. p. 1321-9.

[27] Aldén M, Schawlow AL, Svanberg S, Wendt W, Zhang P-L. Opt Lett 1984;9:211.

[28] Bernstein JS. Laser ionization studies of hydrocarbon flames. Ph.D. dissertation, Cornell University, Ithaca, New York, 1991.

[29] Bernstein JS, Fein A, Choi JB, Cool TA, Sausa RC, Howard SL, Locke RJ, Miziolek AW. Combust Flame 1993;92:85.

[30] Fein A, Bernstein JS, Song X-M, Cool TA. Appl Opt 1994;33:4889.

[31] Bertagnolli KE, Lucht RP, Bui-Pham MN. J Appl Phys 1998;83:2315.

[32] Gross KP, McKenzie RL, Logan P. Exp Fluids 1987;5:372.

[33] Seitzman JM, Hanson RK. AIAA J 1993;31:513.

[34] Reisel JR, Partridge Jr. WP, Laurendeau NM. JQSRT 1995;53:165.

[35] Fiechtner GJ, Carter CD, Barlow RS. Proceedings of the 33rd national heat transfer conference. New York, NY: The American Society of Mechanical Engineers, 1999. p. 1-8.

[36] Peticolas WL. Ann Rev Phys Chem 1967;18:233.

[37] Worlock JM. In: Arechi FT, Schulz-Dubois EO editors. Laser handbook, vol. 2. New York; North-Holland 1972. p. 1323.

[38] Lambropoulos P. In: Bates DR editor. Advances in atomic and molecular physics, vol. 12. New York; Academic Press 1976. p. 87.

[39] Wirth MJ, Lytle FE. In: Heiftje GM editor. New applications of lasers to chemistry, New York; American Chemical Society 1978. p. 24.

[40] Andrews DL. Am J Phys 1985;53:1001.

[41] Moscatelli FA. Am J Phys 1986;54:52.

[42] Hilborn RC. Am J Phys 1982;50:982.

[43] Milonni P, Eberly JH. Lasers. New York: Wiley, 1988.

[44] Weber HP. IEEE J Quantum Electron 1971;QE-7:189.

[45] Louden R. The quantum theory of light. New York: Oxford University Press, 1973.

[46] McKenzie RL, Gross KP. Appl Opt 1981;20:2153.

[47] Schubert M, Wilhelmi B. Nonlinear optics and quantum electronics.. New York: Wiley, 1986.

[48] Fisher WG, Partridge Jr. WP, Dees C, Wachter EA. Photochem Photobiol 1997;66:141.

[49] Sanchez F. Nuovo Cimento B 1975;27:305.

[50] Chrostowski J. Opt Acta 1980;27:1401.

[51] Bamford DJ, Hickman AP, Dyer MJ, Bischel WK. J Opt Soc Am B 1988;5:1369.

[52] McIlrath TJ, Hudson R, Aiken A, Wilkerson TD. Two photon LIDAR technique for remote sensing of atomic oxygen. Institute for Physical Science and Technology Technical Note No. BN-856, University of Maryland, College Park, Maryland, 1977.

[53] Burris Jr. JF. A two-photon absorption cross section measurement in the gamma band system of nitric oxide. Ph.D. dissertation, University of Maryland, College Park, Maryland, 1982.

[54] Burris J, McIlrath TJ. J Opt Soc Am B 1985;2:1307.

[55] Burris J, McIlrath TJ. J Opt Soc Am B 1985;2:1313.

[56] Bamford DJ, Jusinski LE, Bischel WK. Phys Rev A 1986;34:185.

[57] Saxon RP, Eichler J. Phys Rev A 1986;34:199.

[58] Bamford DJ, Dyer MJ, Bischel WK. Phys Rev A 1987;36:3497.

[59] Bamford DJ, Saxon RP, Jusinski LE, Buck JD, Bischel WK. Phys Rev A 1988;37:3259.

[60] McCann MP, Chen CH, Payne MG. J Chem Phys 1988;89:5429.

[61] McCann MP, Chen CH, Datskou I, Evans S. Chem Phys 1993;174:417.

[62] Goehlich AG, Kawetzki T, Döbele HF. J Chem Phys 1998;108:9362.

[63] Di Rosa MD, Farrow RL. J Opt Soc Am B, 1999;16:1988. 
[64] Gross KP, McKenzie RL. J Chem Phys 1982;76:5260.

[65] Marx BR, Simmons J, Allen L. J Phys B 1978;11:L273.

[66] Payne MG, Chen CH, Hurst GS, Kramer SD, Garret WR, Pindzola M. Chem Phys Lett 1981;79:142.

[67] Bergström H, Hallstudius H, Lundberg H, Persson A. Chem Phys Lett 1989;155:27.

[68] Pobst JA. Multiphoton laser induced fluorescence for measuring point specific densities of ground state atomic hydrogen in an arcjet plume. Ph.D. dissertation, University of Southern California, Los Angeles, CA, 1997.

[69] Wysong IJ, Pobst JA. App Phys B 1998;67:193.

[70] Lytle FE, Hassinger TL, Johnson MC. Intern J Environ Anal Chem 1980;8:303.

[71] Kennedy SM, Lytle FE. Anal Chem 1986;58:2643.

[72] Jones RD, Callis PR. J Appl Phys 1988;64:4301.

[73] Lakowicz JR, Gryczynski I, Gryczynski Z, Danielsen E, Wirth MJ. J Phys Chem 1992;96:3000.

[74] McClain WM. Acc Chem Res 1974;7:129.

[75] Xu C, Guild J, Webb WW, Denk W. Opt Lett 1995;20:2372.

[76] Fisher WG, Wachter EA, Lytle FE, Armas M, Seaton C. Appl Spectrosc 1998;52:536.

[77] Göppert-Mayer M. Ann Phys 1931;9:273.

[78] Naskrecki R, Ménard M, van der Muelen P, Vigneron G, Pommeret S. Opt Commun 1998;153:32.

[79] Speiser S, Kimel S. Chem Phys Lett 1970;7:19.

[80] Karule E. In: Bates D, Bederson B. editors. Advances in atomic, molecular, and optical physics, vol. 27. New York: Academic Press, 1991. p. 265. 